\title{
The Temporal Structure of Motivation
}

\author{
III. Identification and Ecological Significance of \\ Ultradian Rhythms of Intracranial Reinforcement
}

\author{
RICHARD J. KATZ ${ }^{1}$ \\ Mental Health Research Institute, Department of Psychiatry, \\ University of Michigan Medical Center, \\ Ann Arbor, Michigan 48109
}

\begin{abstract}
Adult male Sprague-Dawley rats were stereotactically implanted with intracranial electrodes aimed at the anterior medial forebrain bundle, and trained to self-administer intracranial stimulation. Following 3 weeks of continuous access to the self-stimulation contingency, response patterns were recorded on a consecutive minute-by-minute basis for power-spectral analysis. Spectral analysis revealed the existence of significant ultradian rhythms of reward. The functional significance of these rhythms was further explored in a subsequent experiment, in which temporally correlated feeding and self-stimulation patterns were demonstrated. These findings demonstrate a continuous relationship of a biological reward and ICS.
\end{abstract}

Previous reports (reviewed elsewhere; see, for example: Hoebel, 1974; Katz, Baldrighi, \& Roth, 1978; Mogenson \& Phillips, 1976) have identified functionally complementary relationships between feeding and intracranial reward (intracranial self-stimulation; ICS) at numerous met- and diencephalic sites. Included among typical effects are increases in ICS after food deprivation and decreases in ICS with satiety (op. cit.). Conversely, feeding may increase supranormally with restriction of ICS access (Katz et al., 1978). Finally, longitudinal studies of free feeding rats with continuous food and ICS access indicate ICS rates are typically above normal on days when feeding is below normal, and below normal during higher than normal food intake (Katz et al., 1978; Experiment 1). Thus, feeding-ICS relationships may be demonstrated by a variety of techniques requiring neither severe nor prolonged deprivation.

1 The design of the feeding measurement apparatus was suggested by Dr. Larry Morin of Dartmouth College. I am grateful to Dr. Morin for generously sharing the details of his design with me, and for suggesting the use of spectral analysis in the first experiment. The technical support of Giulio Baldrighi, statistical assistance of Bob Shea, and editorial assistance of Abe Feingold are also gratefully acknowledged. Dr. Katz is a Sloan Foundation Fellow in Neuroscience. 
The above findings all utilized a dependent variable recorded in intervals ranging from 1 to $24 \mathrm{hr}$. A number of questions requiring shorter measurement intervals have as yet remained uninvestigated. When ICS is chronically available and measured at 30- to 60-sec intervals, "meal-like" episodes with mathematical properties quite similar to normal feeding patterns are present (Katz, 1979). These patterns are not fully characterized as yet, and biological rhythms, although present, have not been identified in detail (Katz, 1980).

The present report had two closely related ends. The first was a more complete characterization of the biological rhythms underlying intracranial self-stimulation. In addition, the temporal relationship of feeding and ICS episodes was examined.

\section{GENERAL MATERIALS AND METHODS}

Subjects. Fifteen adult male Sprague-Dawley rats (Charles River Farms) ranging between 350 and $420 \mathrm{~g}$ free feeding weight were individually housed in standard rack-mounted $25 \times 18 \times 17-\mathrm{cm}$ stainless-steel cages with food (Teklad $4.0 \%$ fat rodent diet S-0836) and tap water continuously available. Seven rats were used for the first experiment and eight in the remaining experiment. Day/night cycles of 12/12 hr (lights on $=0700-1900$ ) were maintained by electronic timers. Masking noise of 30 $\mathrm{db}$ was provided by the continuous operation of air-circulating equipment.

Apparatus. Cages were modified to allow chronic access to ICS, using the design of Wolf, DiCara, and Simpson (1973). Home cages were modified to house an overhead mounted $14 \times 16-\mathrm{cm}$ stainless-steel plate $14 \mathrm{~cm}$ from the cage floor. Upward displacement of the plate by the head-mounted contact for stimulation (taken from a suede brush) resulted in the closure of a microswitch with a $25-\mathrm{g}$ operating requirement. Circuit completion resulted in the delivery through the plate of a $0.3-\mathrm{sec}$ train of $60 \mathrm{cps}$ sinusoidal current ranging between 10 and $50 \mu \mathrm{A}$ with the cage floor serving as the return circuit for the stimulation. A series of capacitors, a $50-\mathrm{k} \Omega$ resistance, and a zero-crossing relay in series with the stimulator insured constant current conditions across stimulation trains.

One further cage modification was employed for the second experiment. To allow measurement of feeding, normal access to the food hopper was impeded by a $14 \times 18-\mathrm{cm}$ Plexiglas shield containing an $8.5 \times 5-\mathrm{cm}$ aperture $4 \mathrm{~cm}$ from the shield base. Perpendicular sides extended from the shield $6 \mathrm{~cm}$ into the cage, effectively limiting access to the single aperture. A pivoted elevated floor was mounted in front of the access point and rested above a microswitch activated by a 100 -g operating requirement (Fig. 1). Time of approach to the hopper and feeding offset were recorded based upon switch closures and release. It should be noted that the apparatus did not yield a direct measure of intake, however based upon informal visual observation, the $4-\mathrm{cm}$ height and 5 -cm width of the approach avenue effectively discouraged non-food-related entries. 


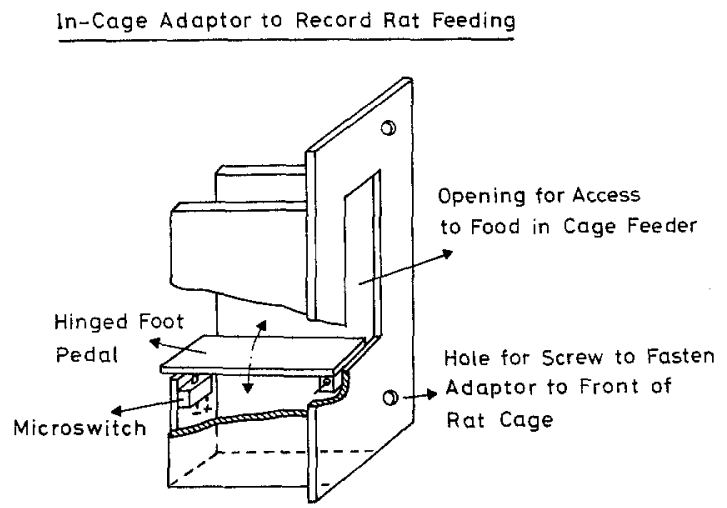

FIG. 1. Feeding apparatus as employed in second experiment. Apparatus is mounted within the self-stimulation cage and effectively limits all approaches to the food hopper. Idea for the hopper courtesy of Dr. Larry Morin (personal communication).

Surgery. Rats were anesthetized with $50 \mathrm{mg} / \mathrm{kg}$ of sodium pentobarbital, administered intraperitoneally. The brush-intracranial electrode assembly, as described in $\operatorname{Katz}(1979,1980)$ was stereotactically implanted, and attached to the skull with five stainless-steel screws and acrylic dental cement. Implant coordinates (in $\mathrm{mm}$ ) from the Bregma suture using a level skull were $0.0,-2.0,-8.0$ (posterior, lateral, and ventral, respectively). One week was allowed for recovery.

Site of the implant was verified in half of the rats in each experiment by the method of Hosko (1975) as modified in our laboratory. At the close of testing, rats were sacrificed with an overdose of sodium pentobarbital and perfused with $0.9 \%$ sodium chloride followed by formalin-acetic acidalcohol fixative (Luna, 1960). Brains were sectioned cryostatically at 40 $\mu \mathrm{m}$, with wet tissue used for visualization by a projecting microscope.

Behavioral procedure. Experimental protocols were essentially similar to descriptions in previous reports (Katz et al., 1978; Katz, 1979, 1980). Stimulation at a constant level of $50 \mu \mathrm{A}$ was activated subsequent to surgical recovery. In the succeeding week rats either shaped themselves during normal exploration or were guided to the overhead panel and shaped, with suitable current adjustments to avoid obvious motor artifacts or signs of aversion. For all rats current was increased or decreased to maintain daily rates of $1000-4500$ responses $/ 24 \mathrm{hr}$. This rate compares to an operant baseline rate of less than 40 responses $/ 24 \mathrm{hr}$ when current was not available. By Week 3,99\% of responding was in the normal dark cycle (i.e., the normally active portion of the rat activity cycle) and greater than $99 \%$ of all responses occurred in discrete episodes by criteria established in Katz (1979) and Katz (1980). Experimental observations were carried out during the third week of exposure. It should be noted that stimulation parameters remained unchanged for at least 2 weeks preceding this. 
Statistical analysis. Initial characterization of biological rhythms was by spectral density analysis (Durbin, 1967; Jenkins \& Watts, 1968), a technique which attempts to decompose the total variance of a time series into component sine curves and a residual (i.e., noncyclic) variance, weighed for their contributions to the series. The median spectral statistic was used to formally test the null hypothesis of equal contributions of all (including residual) components to the power spectrum. Further tests (Experiment 2) were based upon bivariate correlations, conditional probabilities, and cross-correlations. All analyses were based upon 720 -min records.

\section{EXPERIMENT 1}

In a previous study we noted the presence of underlying biological rhythms in self-stimulation (Katz, 1980). The first experiment was carried out to further identify and characterize these ultradian (i.e., shorter than 24-hr) rhythms.

\section{Materials and Methods}

Subjects, apparatus, and procedures were identical to the descriptions provided in the general methods. Statistical analysis also followed previous descriptions.

\section{Results}

Prominent biological rhythms were found in all subjects and their presence was confirmed as being statistically reliable beyond chance. Table 1 provides a synopsis of the behaviors which were further analyzed. It is evident that individual subjects typically responded in discrete episodes. Number of episodes varied between 20 and 60 per night, depending on the subject. Episode length varied slightly from a median value of $2.9 \mathrm{~min}$, while rate of response within episodes was between approximately 17 and

TABLE 1

Response Characteristics of Individual Rats Upon Which Time

Series Analysis Was Subsequently Carried Out ${ }^{a}$

\begin{tabular}{lccc}
\hline $\begin{array}{l}\text { Rat } \\
\text { number }\end{array}$ & $\begin{array}{c}\text { Episodes/ } \\
12 \mathrm{hr}\end{array}$ & $\begin{array}{c}\text { Average episode } \\
\text { length (min) }\end{array}$ & $\begin{array}{c}\text { Average response rate } \\
\text { (Running rate)/episode }\end{array}$ \\
\hline 1 & 61 & 2.6 & 29.0 \\
2 & 40 & 2.5 & 32.7 \\
3 & 43 & 2.8 & 16.6 \\
4 & 47 & 2.9 & 22.9 \\
5 & 32 & 3.4 & 37.0 \\
6 & 23 & 3.6 & 20.0 \\
7 & 34 & 3.1 & 41.8 \\
\hline
\end{tabular}

${ }^{a}$ Based upon 720 consecutive 1-min intervals during the normal dark cycle. 
40. These values all are within the ranges established by our previous reports (Katz, 1979, 1980).

Table 2 presents the characteristics of spectral density analysis for these subjects. Presentation of rhythms was simplified by rounding to the nearest decimal. Precise frequencies were determined by the use of a Parzen window technique as recommended by Jenkins and Watts (1968). Values are reported to the nearest decimal, however, to allow more succinct data presentation and because additional precision would be spurious given initial measurement intervals no more accurate than 60.0 sec. For individual subjects, although patterns varied considerably, trends of prominent (39-40, 26-27, and 19-20 min) contributions, as well as contributions from briefer ultradian rhythms, were observed. It is also evident that the spectra of all rats were denser in rhythms than in the residual. By the median spectral test all subject's spectra reliably were different from a condition of equal density.

\section{Discussion}

Ultradian rhythms of ICS were demonstrated in the rat. In a previous report we noted the existence of biological rhythms across overnight ICS records. These were not quantified however. The only previous demonstration of any ultradian ICS rhythm was in the rhesus monkey (Maxim \& Storrie, 1979) based upon linear regression analysis. The present findings extend these earlier results to a nonprimate species, a novel ICS paradigm, and a different analytic technique.

Rhythms reported in the first experiment were shorter than those in the monkey (op. cit.) although this would be predicted given both a higher basal metabolic rate and shorter rest-activity cycle of the rat in comparison with the monkey. The rhythms did not correlate with any observable programmed environmental changes, and it is therefore possible that they were endogeneous. The significance of these rhythms requires further investigation.

\section{EXPERIMENT 2}

\section{Significance of Biological Rhythms and Relations to Feeding Behavior}

The first experiment pointed to the existence of ultradian rhythms of ICS. Since no external environmental changes were identified which readily correlated with these rhythms, and since spectral density loadings of different rhythms varied idiosyncratically across subjects, it may be argued they reflected endogenous ultradian properties of the reward system.

It has already been noted that feeding and ICS have similar properties both at molar and at more detailed levels of behavioral analysis. Experiment 2 examined the existence of more direct relationships at the level of individual response episodes. If feeding and ICS episodes were directly 


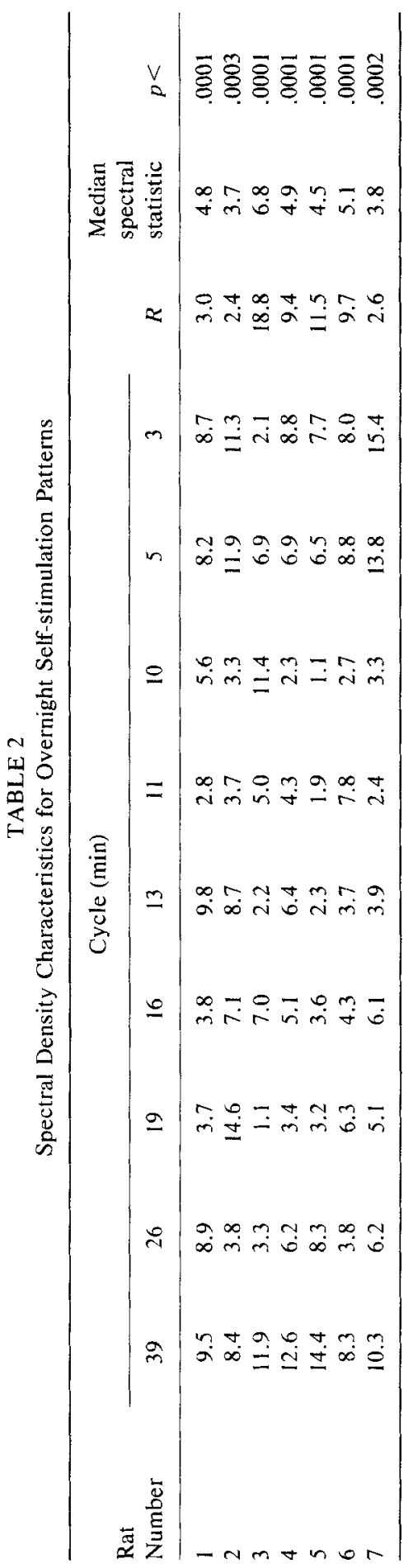


related it would be predicted that (a) numbers of episodes of both feeding and ICS should correlate, (b) conditional probabilities of the two events might be greater than absolute individual event probabilities, and (c) their time series should correlate.

\section{Materials and Methods}

Subjects, apparatus, and procedures were identical to descriptions provided in the general methods section. The only change of note between the first and second experiments was the inclusion of a microswitch sensor for the direct monitoring of feeding. Three tests of an episodic relation between feeding and ICS were carried out. Initially, 12-hr sessions were divided into 6-hr blocks (rationale for viewing first and second 6-hr blocks as different is provided in Katz (1980)) and correlations of episodes per half session were calculated. If feeding and self-stimulation are positively related, high correlations should offer direct evidence of this. Second, individual conditional response probabilities were calculated for feeding and ICS across the entire 12-hr session. Conditional probabilities were compared with absolute probabilities of response to see if episodes were conditional upon each other. For purposes of standardization, a criterion interval of no greater than $120 \mathrm{sec}$ between any feeding and ICS episodes was taken to indicate a positive conditional probability. Any episodes occurring more than $120 \mathrm{sec}$ from each other were considered unrelated. Finally, the time series technique of crosscorrelational analysis was used to determine if the two series of episodes were reliably related across the 12 -hr session.

Results

All three tests of an episode by episode relation of feeding and ICS were positive. Figure 2 provides a scatter plot showing a high positive correla-

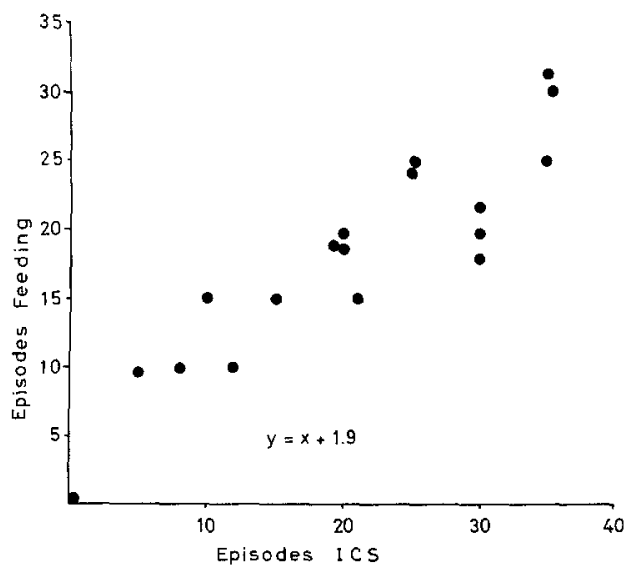

Fig. 2. Half session correlations of feeding and ICS episodes for individual subjects. 
tion between feeding and ICS over consecutive 6-hr epochs. The regression coefficient of .82 was statistically significant beyond chance $(t(14)=$ $5.4, p<.0001$ ) by Fisher's transformation (Bruning \& Kintz, 1977). Thus, at a static level the episodes appeared to share at least a molar level of relationship. The episodes also were significantly contingent upon each other (Table 3). It is evident that when individual subjects are examined there was a definite positive relationship between feeding and ICS (mean values of coefficients for the eight rats $( \pm$ SEM) were $.79 \pm .03 ; .04 \pm .01$; $.04 \pm .01$; and $.77 \pm .04$ for the probabilities of feeding given ICS, absolute feeding episode probability, absolute ICS episode probability, and ICS probability given feeding, respectively). Thus ICS was typically followed by feeding. To further illustrate the major relationship of feeding and ICS, Fig. 3 presents an individual record of responding illustrating typical associations between episodes.

Finally, cross-correlations of the two series were carried out based upon the same maximal lag criteria as above. The respective crosscorrelation coefficients of the eight rats, in the same order as Table 3 were: $.12, .14, .18, .11, .10 ; .10, .09, .16$. Based upon $d f=719$ and Fisher's transformation to $z$, all correlations were significant $(z=3.1,3.8$, $4.1,2.9,2.7,2.7,2.4,4.3 ; p<.05$ in all cases). Although significant relationships across series were established, their low absolute level of relationship requires further explanation and will be commented on below. Histology (Fig. 4) indicated electrodes were localized in the intended site of inplication at the anterior aspect of the medial forebrain bundle.

\section{Discussion}

All tests of an episodic relationship between feeding and ICS were positive. This is most strongly seen in the correlations of episodes over

TABLE 3

Absolute and Conditional Probabilities of Feeding and

Self-Stimulation Epsiodes per $12 \mathrm{hr}(n=8)$

\begin{tabular}{lcccc}
\hline $\begin{array}{c}\text { Rat } \\
\text { number }\end{array}$ & $\begin{array}{c}\text { Probability } \\
\text { of feeding } \\
\text { given ICS }\end{array}$ & $\begin{array}{c}\text { Probability } \\
\text { of feeding } \\
\text { (absolute } \\
\text { per session) }\end{array}$ & $\begin{array}{c}\text { Probability } \\
\text { of ICS } \\
\text { (absolute } \\
\text { per session) }\end{array}$ & $\begin{array}{c}\text { Probability } \\
\text { of ICS } \\
\text { given feeding }\end{array}$ \\
\hline 1 & .90 & .05 & .05 & .90 \\
2 & .64 & .05 & .06 & .76 \\
3 & .87 & .06 & .07 & .87 \\
4 & .75 & .07 & .07 & .75 \\
5 & .82 & .03 & .03 & .82 \\
6 & .66 & .07 & .07 & .66 \\
7 & .78 & .02 & .03 & .52 \\
8 & .93 & .03 & .03 & .93 \\
\hline
\end{tabular}

${ }^{a}$ In all cases $p<.01$ in comparison with absolute probabilities based upon Fishers' transformation to $z$ (Bruning \& Kintz, 1977). 


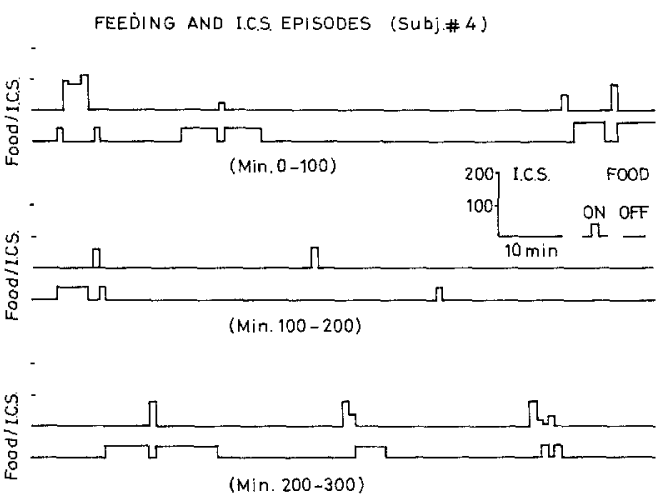

FIG. 3. Individual record of a rat with chronic access to self-stimulation and food (first 300 min of overnight session). It may be seen that there is a close correspondence between ICS and the initiation of feeding. To simplify presentation feeding switch closures are preserved as binary data.

half-nightly sessions and conditional probabilities across $12 \mathrm{hr}$. Baldwin and Parrott (1979) have reported essentially similar findings for episodic feeding by ICS correlations across time however this was in pigs, and with a different time scale. In addition, in the present report series-by-series cross-correlations were statistically significant beyond chance, but they were not strikingly positive. This last analysis therefore afforded only limited support for the hypothesis.

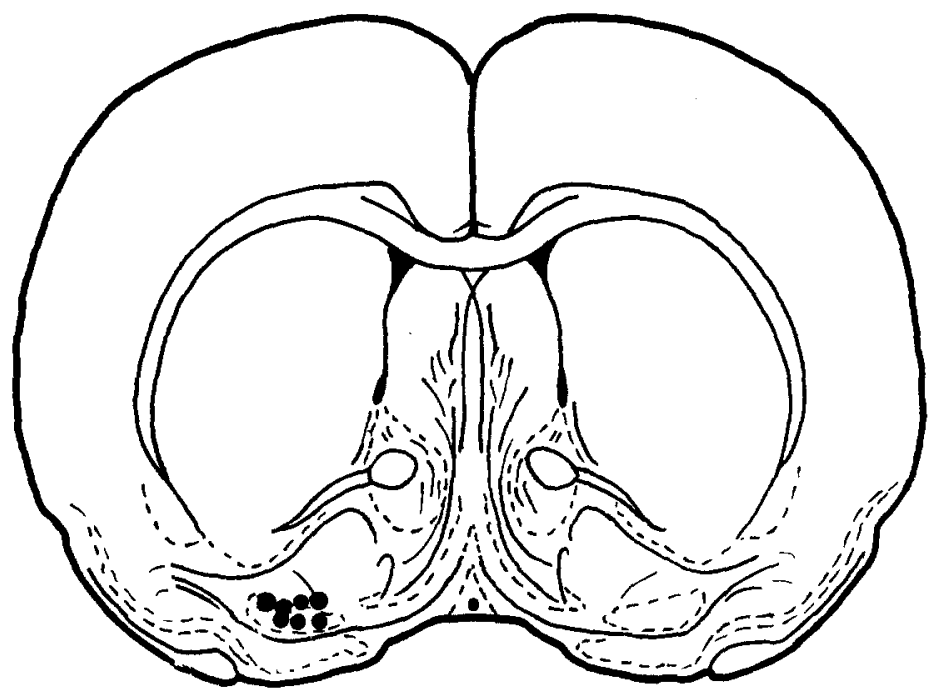

FIG. 4. Composite histology for rats from both experiment sites was visualized from wet-mounted $40 \mu \mathrm{m}$ tissue sections as described in detail in text. 
It must be noted that there may be a number of reasons for low cross-correlations. ICS is clearly ordered upon a ratio scale, i.e., higher positive values are directly related to amounts of ICS. Switch closures for feeding are less clearly ordered. While they are nominal, in that some responding is taken to indicate feeding, they are not strictly ordinal beyond the 0,1 interval i.e., it is not clear that higher per minute counts necessarily are correlated with greater feeding. This problem in scaling may introduce sufficient randomness into the system to lower otherwise present correlations. That correlations were significant despite these problems may speak to a fairly robust relationship requiring different measures for a more accurate measurement.

\section{GENERAL DISCUSSION}

The present findings suggest that the previous reports by ourselves and others (see above) upon molar relationships of feeding and ICS extend to a minute-by-minute relationship under steady states. Quite possibly the immediate activation of feeding and ICS renders complementary increases in ICS and feeding more likely. One activity may "prime" another. Priming is known to play a role in ICS episodes (Katz, 1980) and such effects may be more general. Others have noted that ICS priming may reduce the likelihood of feeding, however (e.g., Stellar \& Heard, 1976). These apparently discrepant data may nonetheless be consistent with the present view. It has already been demonstrated that individual ICS episodes are characterized by high initial priming effects which decrease during an episode until the relative ineffectiveness of priming terminates them (Katz, 1980). Thus, in the present design, it may not be a direct ICS priming effect, but the postpriming interval which controls feeding. If postpriming processes were responsible for increased feeding, then it would be hypothesized that priming might facilitate feeding if a sufficiently long interval followed a train of priming which itself was sufficient in duration to cause habituation of its immediate motivational effects. Experiments on the issue have not been carried out, but may offer a possible reconciliation of two divergent sets of data.

. The above explanation presupposes a specific association between the aftereffects of ICS and the initiation of feeding. An alternative explanation might involve increases in general activity at the termination of a stimulation episode. By this second explanation the observed specificity of ICS and feeding might reflect limitations of response measurement. Possibly if drinking, gnawing, or general motor activity were measured, they would show increases of the same magnitude as feeding. Such an interpretation clearly would vitiate an account based upon a motivationally specific aftereffect, and favor a more plastic view of postpriming processes. Indirect evidence in favor of plasticity may be found in the studies of Gallistel and co-workers (note in particular Gallistel, 1966; Stellar \& Gallistel, 1975). 
Although no direct test of postpriming specificity was incorporated into the present design several factors favor its presence to some degree. For example, despite the fact that in other circumstances both feeding and drinking may be spontaneously elicited from the same electrode, both responses may be specific in the sense they are highly dependent upon stimulation frequency (Mogenson, Gentil, \& Stevenson, 1971). In fact a given site at a given frequency might exclusively yield only one behavior. Similarly, it is possible to dissociate rewarding and general activating effects of brain stimulation by parametric manipulation (e.g., Miliaressis \& LeMoal, 1976; Rompre \& Miliaressis, 1980). It seems implausible, especially given constant current stimulation conditions as employed in the present design that stimulation parameters maximizing general activities were fortuitously present in all rats and to the same degree. Moreover access to the food hopper was obstructed in a way so as to impede all but deliberate approach attempts demanding some effort on the part of the subject. It is unlikely that a nonspecifically activated rat would choose a difficult approach, given the presence of less demanding behavioral alternatives. Thus the effort demanded by the approach also indirectly agrees with the induction of a fairly specific motivational state.

In conclusion, it is clear that relationships across the motivational systems of feeding and ICS may be investigated with sufficient parametric generality to allow the framing of general hypotheses of interrelationships. From previous and present studies it may be seen that relationships may be traced and analyzed across measurement intervals varying by a factor of almost 10,000 and through a number of different techniques. Despite changes to scale and procedure, similar behavioral relationships may nonetheless be demonstrated.

Note added in proof. DePaulo and Hoffman (1980) in their elegant examination of filial behaviors in ducklings noted that the termination of bursts of social responding was associated with increased grooming and activity. This may point to motivational parallels to the present results, however it must be kept in mind that the above study also noted a more common pattern of spontaneous burst cessation without a clear behavioral aftereffect.

\section{REFERENCES}

Baldwin, B. A., \& Parrott, R. F. (1979). Studies on intracranial electrical self-stimulation in pigs in relation to ingestive and exploratory behavior. Physiology and Behavior, 22, 723-730.

Bruning, J. L., \& Kintz, B. L. (1977). Computational Handbook of Statistics, 2nd ed., Glenview, Ill.: Scott, Foresman.

DePaulo, P., \& Hoffman, H. S. (1980). The temporal pattern of attachment behavior in the context of imprinting. Behavioral and Neural Biology, 28, 48-64.

Durbin, J. (1967). Tests of serial independence based on the cumulative periodogram. Bulletin of the International Statistics Institute, 44, 1-9.

Gallistel, C. R. (1966). Motivating effects in self-stimulation. Journal of Comparative and Physiological Psychology, 69, 713-721.

Hoebel, B. G. (1974). Brain reward and aversion systems in the control of feeding and sexual behavior. Nebraska Symposium on Motivation, 22, 48-112. 
Hosko, M. J. (1975). Technique for rapid permanent documentation of intracerebral electrode sites. Physiology and Behavior, 14, 367-368.

Jenkins, G. M., \& Watts, D. G. (1968). Spectral Analysis and its Application. San Francisco: Holden-Day.

Katz, R. J., Baldrighi, G., \& Roth, K. A. (1978). Appetitive determinants of self-stimulation. Behavioral Biology, 22, 500-508.

Katz, R. J. (1979). The temporal structure of reinforcement: an analysis of brain stimulated reward. Behavioral and Neural Biology, 26, 416-430.

Katz, R. J. (1980). The temporal structure of motivation. II. Determinants of reinforcement patterns in local organization of intracranial reward. Behavioral and Neural Biology, 28, 463-472.

Luna, L. G. (1960). Manual of Histological Staining Methods of the Armed Forces Institute of Pathology. New York: McGraw-Hill.

Maxim, P. E., \& Storrie, M. (1979). Ultradian barpressing for rewarding brain stimulation in rhesus monkeys. Physiology and Behavior, 22, 683-697.

Miliaressis, E., \& LeMoal, M. (1976). Stimulation of the medial forebrain bundle: behavioral dissociation of its rewarding and activating effects. Neuroscience Letters, 2, 295-300.

Mogenson, G. J., Gentil, C. G., \& Stevenson, J. A. F. (1971). Feeding and drinking elicited by low and high frequencies of hypothalamic stimulation. Brain Research, 33, 127-137.

Mogenson, G. J., \& Phillips, A. G. (1976). Motivation: a psychological construct in search of a physiological substrate. Progress in Pyschobiology and Physiological Psychology, 6, 189-243.

Rompre, P. P., \& Miliaressis, E. (1980). A comparison of the excitability cycles of the hypothalamic fibers involved in self-stimulation and exploration. Physiology and $B e-$ havior, 24, 995-998.

Stellar, J. R., \& Gallistel, C. R. (1975). Runway performance of rats for brain stimulation or food reward: effects of hunger and priming. Journal of Comparative and Physiological Psychology, 89, 590-599.

Stellar, J. R., \& Heard, K. (1976). Aftereffects of rewarding lateral hypothalamic brain stimulation and feeding behavior. Physiology and Behavior, 17, 865-867.

Wolf, G., DiCara, I. V., \& Simpson, W. (1973). The contact method: a simple technique for electrical self-stimulation without external leads. Physiology and Behavior, 11, 721723. 